\title{
Analysis of muscle fiber conduction velocity enables reliable detection of surface EMG crosstalk during detection of nociceptive withdrawal reflexes
}

\author{
Michael Brun Jensen*, José Alberto Biurrun Manresa, Ken Steffen Frahm and Ole Kæseler Andersen
}

\begin{abstract}
Background: The nociceptive withdrawal reflex (NWR) is a polysynaptic spinal reflex that induces complex muscle synergies to withdraw a limb from a potential noxious stimulus. Several studies indicate that assessment of the NWR is a valuable objective tool in relation to investigation of various pain conditions. However, existing methodologies for NWR assessment evaluate standard surface electromyography (SEMG) measured over just one muscle and do not consider the possible interference of crosstalk originating from adjacent active muscles. The present study had two aims: firstly, to investigate to which extent the presence of crosstalk may affect NWR detection using a standardized scoring criterion (interval peak z-score) that has been validated without taking crosstalk into consideration. Secondly, to investigate whether estimation of muscle fiber conduction velocity can help identifying the propagating and non-propagating nature of genuine reflexes and crosstalk respectively, thus allowing a more valid assessment of the NWR.

Results: Evaluation of interval peak z-score did apparently allow reflex detection with high sensitivity and specificity (0.96), but only if the influence of crosstalk was ignored. Distinction between genuine reflexes and crosstalk revealed that evaluation of interval peak z-score incorporating a z-score threshold of 12 was associated with poor reflex detection specificity $(0.26-0.62)$ due to the presence of crosstalk. Two different standardized methods for estimation of muscle fiber conduction velocity were employed to demonstrate that significantly different muscle fiber conduction velocities may be estimated during genuine reflexes and crosstalk, respectively. This discriminative feature was used to develop and evaluate a novel methodology for reflex detection from SEMG that is robust with respect to crosstalk. Application of this conduction velocity analysis (CVA) entailed reflex detection with excellent sensitivity (1.00 and 1.00) and specificity (1.00 and 0.96) for the tibialis anterior and soleus muscles.

Conclusion: This study investigated the negative effect of electrical crosstalk during reflex detection and revealed that the use of a previously validated scoring criterion may result in poor specificity due to crosstalk. The excellent performance of the developed methodology in the presence of crosstalk shows that assessment of muscle fiber conduction velocity allows reliable detection of EMG crosstalk during reflex detection.
\end{abstract}

Keywords: Nociceptive withdrawal reflex (NWR), Reflex detection, Surface electromyography (sEMG), Crosstalk, Muscle fiber conduction velocity

\footnotetext{
* Correspondence: mbj@hst.aau.dk

Integrative Neuroscience group, Center for Sensory-Motor Interaction,

Department of Health Science and Technology, Aalborg University, Fredrik

Bajers Vej 7, E1-102, 9220, Aalborg, Denmark
} 


\section{Background}

The nociceptive withdrawal reflex (NWR) is a polysynaptic spinal reflex that induces complex muscle synergies to withdraw a limb from potential noxious stimuli [1]. It can be elicited by percutaneous electrical stimulation of a nerve trunk or free nerve endings located in the skin, and the resulting reflex response is often measured using standard surface electromyography (sEMG) [1,2]. Several studies indicate that assessment of the NWR is a valuable objective tool in relation to assessment of various pain conditions, which may be caused or sustained by neural hypersensitivity with a central component [3].

Several attempts have been made for standardization of NWR assessment methodologies, in order to use them as outcome measures in clinical settings. Consequently, a wide variety of definitions for NWR detection from sEMG signals has been proposed, some of which have demonstrated high accuracy and reliability [4,5]. Nevertheless, existing methodologies for NWR assessment evaluate standard sEMG measured over just one muscle and do not consider the possible interference of crosstalk originating from adjacent active muscles $[1,4,5]$. Electrophysiological signals are conducted through the human tissues and crosstalk may erroneously cause significant sEMG activity to be recorded over an inactive muscle [6]. As a result, some apparent reflexes may reflect nothing but crosstalk.

Crosstalk is one of the main concerns of sEMG recording, and several methods have been proposed to reduce these misleading signal components $[7,8]$. Unfortunately, neither high-pass temporal or spatial filtering nor crosscorrelation of signals detected over different muscles has resulted in a reliable method for reduction or estimation of crosstalk $[9,10]$. However, it has been demonstrated that signals generated by superficial motor units of the active muscle monitored are propagating along the muscle fibers, whereas crosstalk signals are non-propagating [10]. Thus, identification of crosstalk may possibly be based on assessment of whether the signal is dominated by propagating or non-propagating signal components.

The present study had two aims: firstly, to investigate to which extent the presence of crosstalk may affect NWR detection using a standardized scoring criterion [4] that was validated without taking crosstalk into consideration. Secondly, to investigate whether estimation of muscle fiber conduction velocity $(\mathrm{CV})$ can identify the propagating and non-propagating nature of genuine reflexes and crosstalk respectively, thus allowing a more valid assessment of the NWR. More specifically, it was examined if estimations of muscle fiber $\mathrm{CV}$ were higher for crosstalk than for genuine reflexes in the lower extremities of humans. The discriminative value of features extracted using a simple cross-correlation technique (including CV) was analyzed and a novel cross-correlation based $\mathrm{CV}$ analysis (CVA) for NWR detection with improved specificity is presented.

\section{Methods \\ Participants}

Fourteen male volunteers (mean age 24.4 years, range 19-28 years) participated in the study. Written informed consent was obtained from all subjects prior to participation and the Declaration of Helsinki was respected. The study was approved by the local ethical committee ('Den Videnskabsetiske Komité for Region Nordjylland') with approval number VN2005/2.

\section{Electrical stimulation}

Two surface stimulation electrodes $(20 \times 15 \mathrm{~mm}$, type 700, Ambu, Denmark) were mounted on the plantar side of the foot to elicit the NWR, as seen in Figure 1. One large common anode $(100 \times 140 \mathrm{~mm}$, Pals, Axelgaard, USA) was placed on the dorsum of the foot to ensure that the stimulus was perceived as coming from the sole of the foot. The stimulation electrodes were moved slightly in case the evoked sensation indicated direct nerve trunk stimulation (i.e. radiating sensation). Each stimulus consisted of a constant current pulse train of five individual $1 \mathrm{~ms}$ pulses delivered at $200 \mathrm{~Hz}$ (felt as a single stimulus) by a computer controlled electrical stimulator (Noxitest IES 230, Aalborg, Denmark). The stimulation intensity was set as 1.2-1.5 times the initial NWR threshold for each electrode. The two stimulation sites were stimulated in a blinded random sequence with an inter-stimulus interval between 10 and $15 \mathrm{~s}$.

\section{EMG recordings}

Activity in the ipsilateral tibialis anterior (TA) and soleus (SOL) (lateral side) was measured using single-channel and multi-channel sEMG and also intramuscular electromyography (iEMG), see Figure 1 for complete setup.

\section{Single-channel sEMG}

Three surface electrodes (type 720, Ambu, Denmark) were placed in parallel along the overall orientation of the two muscles with an interelectrode distance of $2 \mathrm{~cm}$. A common reference electrode $(100 \times 140 \mathrm{~mm}$, Pals, Axelgaard, USA) was placed on the ipsilateral knee. The tri-polar electrode configuration fed three separate amplifiers for simultaneous recording of two single differential (SD) and one double differential (DD) sEMG signal from each of the two muscles, see Figure 1. The signals were amplified, filtered (5-500 Hz), sampled $(2 \mathrm{kHz})$ and stored (1 s window including $200 \mathrm{~ms}$ pre-stimulation). The signals were interpolated to $10 \mathrm{kHz}$ using an antialiasing, linear-phase, low-pass, finite impulse response filter implemented in Matlab (MathWorks, USA). 


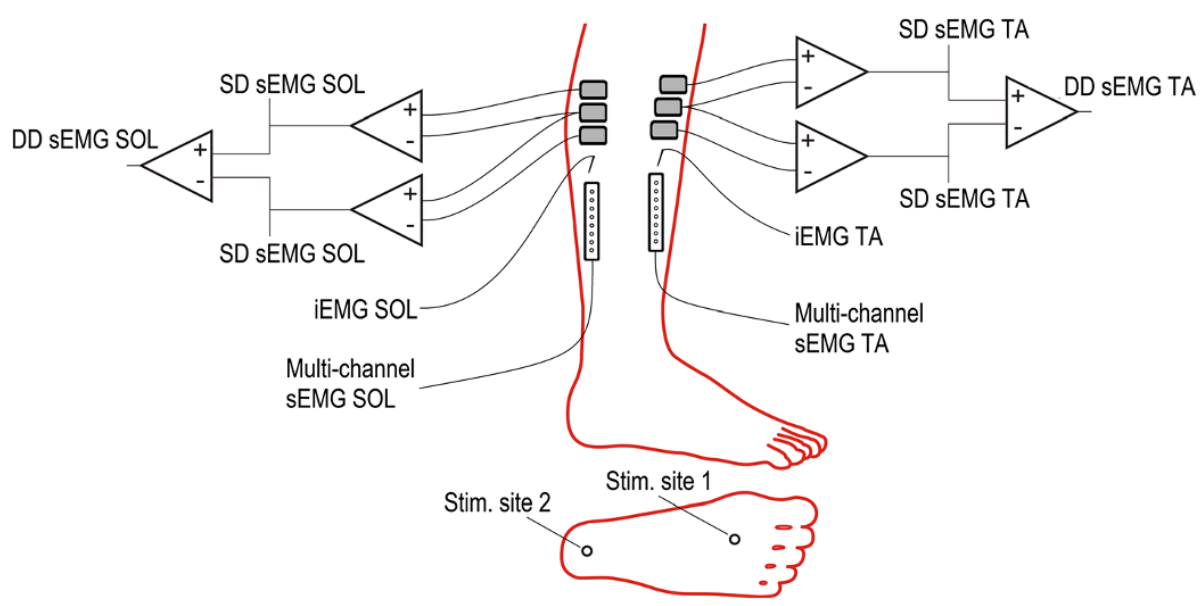

Figure 1 Experimental setup. Single differential (SD) and double differential (DD) surface electromyography (sEMG) and intramuscular electromyography (iEMG) were measured from the tibialis anterior (TA) and soleus (SOL) muscles during reflex responses elicited by electrical stimulation of two sites under the sole of the foot.

\section{Multi-channel sEMG}

Multi-channel sEMG signals were detected with two linear adhesive arrays (type ELSCH008, Spes Medica, Italy), each consisting of eight electrodes with $5 \mathrm{~mm}$ interelectrode distance in bipolar configuration. The adhesive arrays were placed along the overall orientation of the muscles just proximal to the distal tendon. The signals were amplified (EMG-USB2, OT Bioelettronica, Torino, Italy), filtered (10-500 Hz) and sampled at $10 \mathrm{kHz}$.

\section{iEMG}

iEMG signals were recorded using wire electrodes made of teflon-coated stainless-steel (50 $\mu \mathrm{m}$ diameter, A-M Systems, Inc. Carlsborg, USA) with $5 \mathrm{~mm}$ un-insulated tips in bipolar configuration $[11,12]$. The skin was cleaned with alcohol at the insertion points whereupon wire electrodes were placed inside each of the two muscles using a concentric hypodermic needle (25 gauge, $25 \mathrm{~mm}$ ). The signals were amplified (EMGUSB2, OT Bioelettronica, Torino, Italy), filtered $(10-500 \mathrm{~Hz})$ and sampled at $10 \mathrm{kHz}$.

\section{Experimental procedure}

Before mounting the recording and stimulation electrodes, thick epidermal layers on the sole of the foot were ground off using a callus remover and the skin was scrubbed with an abrasive paste in order to reduce skin impedance. The skin above the two muscles was shaved and slightly abraded with an abrasive paste. The subject was sitting relaxed on a chair with the hip, knee and ankle flexed 90 degrees. The subject was thoroughly familiarized with electrical stimulation before actual data acquisition. The NWR threshold was identified for each of the two stimulation sites as the lowest intensity eliciting at least two reflexes in three consecutive stimulations. The subjects were stimulated until ten unambiguous reflexes (see Identification of genuine reflexes) had been recorded from both the TA (stimulation in the arch of the foot) and SOL (stimulation at the heel) muscles. If elicitation of reflexes ceased at a site during the experiment e.g. due to habituation (no reflex elicited by three consecutive stimulations of one site), the NWR threshold for that stimulation site was re-assessed and an adjusted stimulation intensity was applied. If the stimulation intensities became intolerable to the subject the experiment was discontinued.

\section{Data analysis}

\section{Identification of genuine reflexes}

During data acquisition, the recorded signals were visually examined for online identification of NWR using a set of fixed criteria proposed by Rhudy and France [4], i.e., a reflex was identified if at least one sizable difference peak occurred in the reflex window (defined as the 80-150 ms post-stimulus interval), relative to baseline, but not if EMG activity in the reflex window mimicked baseline, see Figure 2. Additional criteria involving the simultaneous assessment of sEMG and iEMG signals were enforced post acquisition to ensure a homogenous dataset, consisting of sweeps containing a genuine reflex in only one of the two antagonistic muscles for data analysis.

The inclusion and exclusion criteria can be summarized as follows. Inclusion: (1) Reflex identified in all sEMG recordings and a simultaneous reflecting burst of iEMG activity recorded from the same muscle (any concurrent sEMG activity measured over the antagonistic muscle was considered crosstalk), see Figure 2. Exclusion: (1) EMG activity in a $200 \mathrm{~ms}$ prestimulus window. (2) sEMG activity not synchronized with iEMG recordings from one of the 


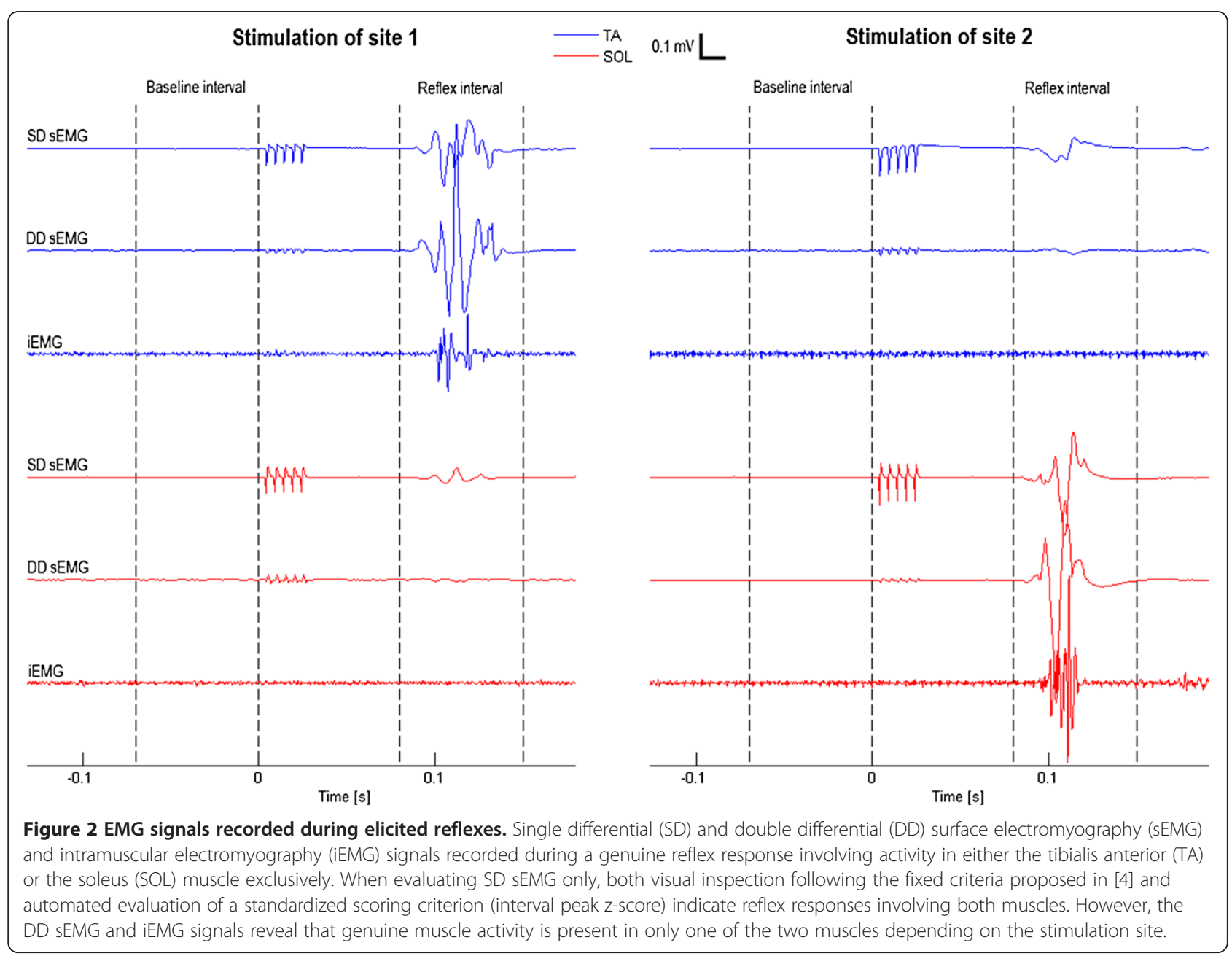

two muscles. (3) iEMG activity in both muscles within the reflex window (co-contraction).

The amplitude of the NWR in the retained sweeps was quantified as the average root mean square amplitude of the two SD signals calculated within in the reflex window. The amplitude of crosstalk was calculated in the same way, based on the two SD signals recorded over the non-active muscle.

\section{Estimation of muscle fiber conduction velocity}

Muscle fiber CV was estimated from multi-channel sEMG recordings of sweeps using a maximum likelihood estimator [13]. In order to avoid unreliable CV estimations, only recordings with a signal-to-noise ratio (SNR) larger than $7 \mathrm{~dB}$ were included in the analysis. Additionally, a simple cross-correlation technique was applied on the standard single-channel sEMG signals [14,15]. For each muscle, the cross-correlation between the proximal and distal SD
sEMG signals was calculated and the conduction time between the two electrode pairs was estimated as the temporal displacement of the peak in the cross-correlogram. Average CV was calculated as the inter-electrode distance divided by the estimated conduction time. Accurate estimation of average $\mathrm{CV}$ requires undistorted propagation of the signals along the muscle fibers. While this ideal assumption is never completely valid in practice due to the finite length of fibers, violations are particularly prone to occur for signal components with long wavelengths relative to the actual length of the muscle fibers [16]. Hence, prior to performing the cross-correlation, the SD sEMG recorded using single-channel electrodes over TA and SOL were high-pass filtered with cut-off frequencies of $80 \mathrm{~Hz}$ and $100 \mathrm{~Hz}$, respectively.

\section{Reflex detection by evaluation of interval peak z-score}

The SD and DD sEMG signals were rectified and their interval peak z-score [4] was calculated as: 
The literature suggests that signals with an interval peak z-score larger than 12 represent a reflex [5]. The sensitivity (i.e. the ability to detect genuine reflexes) and specificity (i.e. the ability to avoid detecting crosstalk as genuine reflexes) of this approach were investigated. Two different visual examinations of the recorded signals were used for validation, considering sEMG and iEMG signals respectively.

\section{Cross-correlation based CVA for reflex detection}

This novel method for reflex detection involved analysis of cross-correlations between the two SD sEMG signals recorded over each muscle and evaluation of features extracted from the resulting cross-correlograms. The cross-correlations were normalized by the product of the norm of each of the two SD sEMG signals.

The method was designed to determine if a response associated with an interval peak z-score larger than 12 indeed represents a genuine reflex or may be attributed to crosstalk. Hence, it was only applied on recordings where both SD sEMG recordings and the DD sEMG recording were associated with an interval peak z-score larger than 12 (otherwise no reflex was detected). The response were attributed to crosstalk (and no reflex was detected) if both the CV estimated from the crosscorrelation and the maximal value of the normalized cross-correlation were above fixed thresholds (specific for each muscle). The thresholds for $\mathrm{CV}$ and maximal correlation were identified by simultaneous optimization of both sensitivity and specificity based on pooled data from all sweeps, i.e., maximization of the intersection between the sensitivity and specificity planes in Figure 3.

\section{Comparison of methods for reflex detection}

CVA was compared to reflex detection based on evaluation of interval peak z-score calculated for both SD and
DD sEMG signals by evaluation of sensitivity and specificity calculated for each individual subject.

\section{Statistics}

Parametric and non-parametric statistical methods were used whenever data was normally or non-normally distributed, respectively. Mann-Whitney $U$ test was used for comparison of $\mathrm{CV}$, whereas Friedman test was used for paired comparison of both sensitivity and specificity of methods for reflex detection, with Student Newman Keuls for post hoc analysis. Independent t-tests were used for comparison of amplitudes of reflexes and crosstalk, respectively. $\mathrm{P}<0.05$ was considered statistical significant. Results are presented as mean \pm standard error of the mean when the underlying data is normally distributed and as median (lower quartile, upper quartile) when it is not.

\section{Results}

The initial reflex thresholds for stimulation of the arch and heel were $9.5 \pm 1.4 \mathrm{~mA}$ and $14.9 \pm 1.3 \mathrm{~mA}$, entailing initial stimulation intensities of $12.6 \pm 1.6 \mathrm{~mA}$ and $20.4 \pm$ $1.7 \mathrm{~mA}$, respectively. The amplitudes of reflexes elicited in TA $(91.8 \pm 3.9 \mu \mathrm{V})$ were significantly larger than those elicited in SOL $(34.0 \pm 2.3 \mu \mathrm{V})\left(\mathrm{t}_{(487)}=10.76, \mathrm{p}<0.001\right)$ and consequently the amplitudes of crosstalk measured over SOL $(8.2 \pm 0.6 \mu \mathrm{V})$ were significantly larger than those measured over TA $(6.7 \pm 0.3 \mu \mathrm{V})\left(\mathrm{t}_{(487)}=13.47, \mathrm{p}<\right.$ $0.001)$. The mean number of genuine reflexes identified in each subject in TA and SOL was 22.0 (range 5-36) and 12.9 (range 5-23), respectively. In comparison, the mean number of reflexes detected by evaluation of interval peak z-scores (including genuine reflexes and crosstalk erroneously detected as reflexes) was 30.5 and 28.5 in TA and SOL, respectively.
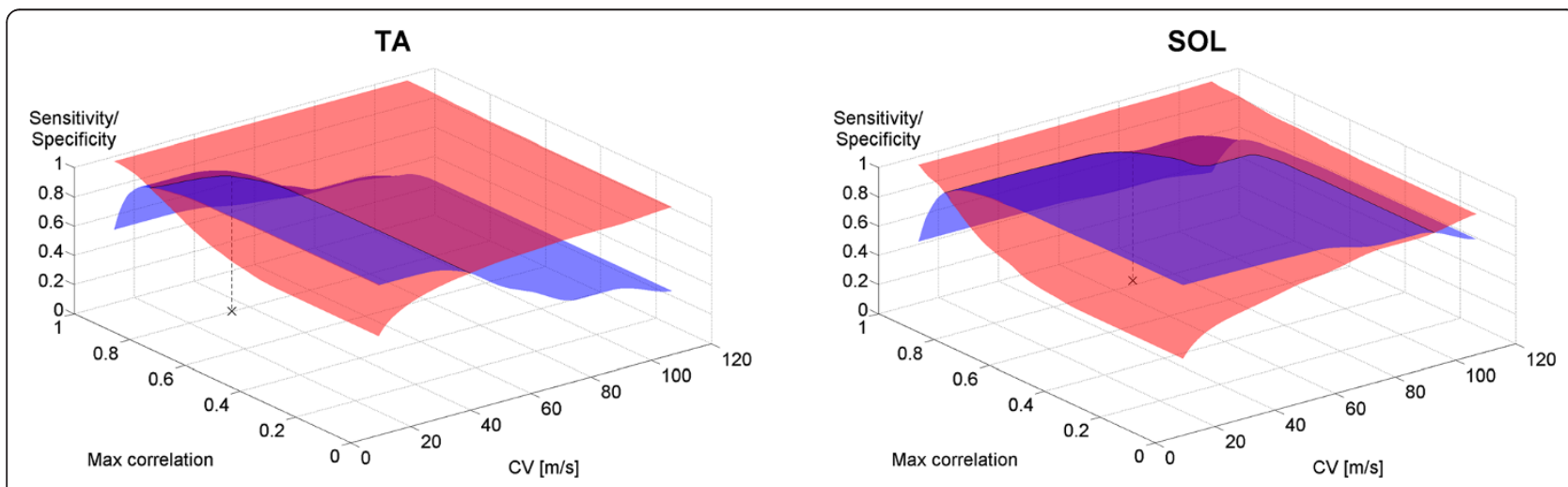

Figure 3 Sensitivity (red/light) and probability (blue/dark) for cross-correlation based conduction velocity analysis (CVA). This novel methodology for reflex detection is based on evaluation of muscle fiber conduction velocity (CV) and maximal cross-correlation estimated from surface electromyography. Sensitivity and specificity is presented for both the tibialis anterior (TA) and soleus (SOL) muscles. The black cross indicates the identified thresholds entailing an equally weighted joint value for sensitivity and specificity of 0.96 and 0.91 for TA and $\mathrm{SOL}$ respectively. 


\section{Muscle fiber conduction velocity}

Due to high selectivity of the multi-channel array electrodes, only 7 and 36 cases of crosstalk (in 308 and 181 recorded reflexes) with a SNR larger than $7 \mathrm{~dB}$ were measured over TA and SOL, respectively. However, muscle fiber CV estimated from both multi-channel sEMG (both muscles, $\left.\mathrm{U}_{(7,36)}=0, \mathrm{z}=4.13, \mathrm{p}<0.001\right)$ and from $\mathrm{sEMG}$ recorded using tri-polar configurations of single-channel electrodes $\left(\mathrm{TA}: \mathrm{U}_{(308,119)}=2603, \mathrm{z}=13.76, \mathrm{p}<0.001\right.$; SOL: $\left.\mathrm{U}_{(181,219)}=2557, \mathrm{z}=15.09, \mathrm{p}<0.001\right)$ clearly demonstrated that crosstalk was associated with significantly higher estimations of muscle fiber $\mathrm{CV}$ than genuine reflexes for both muscles, see Table 1.

\section{Reflex detection by evaluation of interval peak z-score}

The evaluation of interval peak z-score entailed reflex detection with high sensitivity and specificity, when it was validated using solely visual examination of SD sEMG. Moreover, in line with previous studies [4,5], the optimal threshold for the interval peak $\mathrm{z}$-score was 12.6 (at the intersection of sensitivity and specificity curves) assuming equal cost functions for sensitivity and specificity, see Figure 4. In contrast, the use of visual examination of iEMG recordings for validation (allowing distinction between genuine reflexes and crosstalk) indicated that the optimal thresholds for the interval peak z-score were 71 and 48 for evaluation of SD and DD signals respectively, see Figure 4. This comparison furthermore demonstrated that application of an interval peak z-score threshold of 12 was associated with poor reflex detection specificity $(0.26$ and 0.62 respectively) for SD and DD signals.

\section{Cross-correlation based CVA for reflex detection}

The thresholds for CV for TA and SOL were $34 \mathrm{~m} / \mathrm{s}$ and $68 \mathrm{~m} / \mathrm{s}$, whereas the thresholds for maximal crosscorrelation were 0.80 and 0.82 , respectively. These thresholds are indicated in the sensitivity and specificity plots in Figure 3, which illustrates the discriminative power of muscle fiber $\mathrm{CV}$. Evaluation of $\mathrm{CV}$ alone (intersection with the CV-axes in Figure 3) allows an equallyweighted joint value of sensitivity and specificity of 0.90 for both TA and SOL. Moreover, adding the maximal

Table 1 Muscle fiber conduction velocity (CV) estimated from multi-channel surface electromyography (sEMG) using a maximum likelihood estimator [13] and from two single differential sEMG recordings using a simple crosscorrelation technique $[14,15]$, respectively

\begin{tabular}{cccc}
\hline CV $[\mathbf{m} / \mathbf{s}]$ & & Reflex: & Crosstalk: \\
\hline Maximum likelihood & TA: & $6.0(5.0-7.1)$ & $168.7(93.0-198.3)$ \\
estimator & SOL: & $7.8(6.8-9.9)$ & $90.1(53.6-146.5)$ \\
\hline Simple cross-correlation & TA: & $5.3(2.8-14.3)$ & $66.7(50.0-100.0)$ \\
technique & SOL: & $8.7(4.2-40.0)$ & $200.0(100.0-200.0)$ \\
\hline
\end{tabular}

cross-correlation feature results in an increase in joint sensitivity and specificity to 0.96 and 0.91 for TA and SOL, respectively.

\section{Comparison of methods for reflex detection}

CVA showed a significant increase in specificity compared to reflex detection by evaluation of interval peak z-score performed on both SD and DD sEMG signals, see Figure 5. However, reflex detection performed on SD sEMG had significantly lower specificity than detection performed on DD sEMG (TA: $x_{(2)}^{2}=26.08, \mathrm{p}<0.001$; SOL: $x_{(2)}^{2}=25.56, \mathrm{p}<0.001$; all post hoc pairwise comparisons: $\mathrm{p}<0.05)$. A significant difference was identified regarding the sensitivity of the three detection methods, meaning that they are not likely to present the same median sensitivity (TA: $x_{(2)}^{2}=10.00, \mathrm{p}=0.007$; SOL: $x_{(2)}^{2}=$ 9.50, $\mathrm{p}=0.009)$. However, pairwise significant differences were not found, likely due to lack of power of the post hoc multiple comparison tests (no significant post hoc pairwise comparisons: $\mathrm{p}>0.05$ ).

\section{Discussion}

This study investigated the negative effect of electrical crosstalk during reflex detection and revealed that the use of a previously validated scoring criterion may result in poor specificity in the presence of crosstalk. Two different standardized methods for estimation of muscle fiber CV were employed to demonstrate that significantly different CVs may be estimated during genuine reflexes and crosstalk, respectively. This discriminative feature was used to develop and assess a novel methodology for reflex detection from sEMG that is robust with respect to crosstalk.

\section{Identification of genuine reflexes}

The sEMG and iEMG signals recorded together with the set of fixed criteria described in the Methods section, based on physiological knowledge of the human withdrawal reflex, clearly indicate that the signals regarded as crosstalk are indeed so. This conclusion can mainly be drawn due to temporal observations. Studies in both animals [17-20] and humans [21,22] have demonstrated that nociceptive withdrawal reflexes are modularly organized, meaning that each muscle or group of synergistic muscles has a bounded well-defined and unique cutaneous reflex receptive field (RRF). Noxious stimulation of the skin within the RRF may cause a reflex response involving the related muscles, whereas stimulation outside the RRF has no effect or may even inhibit activity in the same muscles $[18,19,23]$. The RRF is hence defined as the skin area from which a reflex can be evoked, which generally adheres to biomechanical function of the related muscle ensuring efficient withdrawal $[17,19]$. During voluntary contraction, the two antagonistic muscles 

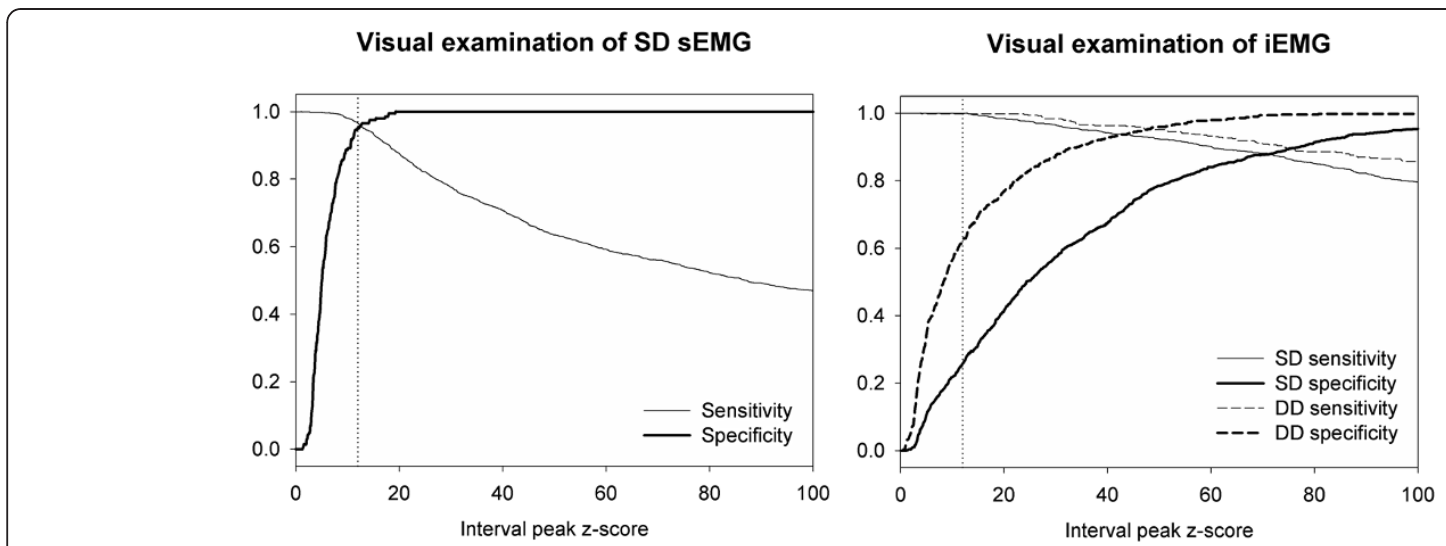

Figure 4 Sensitivity and specificity of reflex detection based on evaluation of interval peak z-scores. The sensitivity and specificity was calculated using two different gold standards; visual examination of single differential (SD) surface electromyography (sEMG) and intramuscular electromyography (iEMG) recordings respectively. The vertical lines represent an interval peak z-score threshold of 12 .

may likely be activated simultaneously (co-contraction) to stabilize a joint. However, due to the functional modular organization of the NWR, this is highly unlikely to occur during a reflex response. A few times during the data acquisition, activity in both muscles were observed within the reflex window. However, these occurrences were never synchronized in the recordings from the two muscles, but could be synchronically identified in both the sEMG and iEMG signals recorded from each of the two muscles, respectively. These recordings were not included in the data analysis, in accordance with the exclusion criteria.

\section{Validation of automated scoring criteria}

Several automated scoring criteria, including the interval peak z-score, have been demonstrated to enable accurate and reliable reflex detection from sEMG signals [4,5]. This comprehensive validation of scoring criteria contributed to the standardization of reflex detection methodology and promoted the evaluation of reflex thresholds as a valuable experimental and clinical tool. However, the validation performed did not consider EMG crosstalk, and the applied gold standard (visual examination of the sEMG signals), did not allow a proper evaluation of the

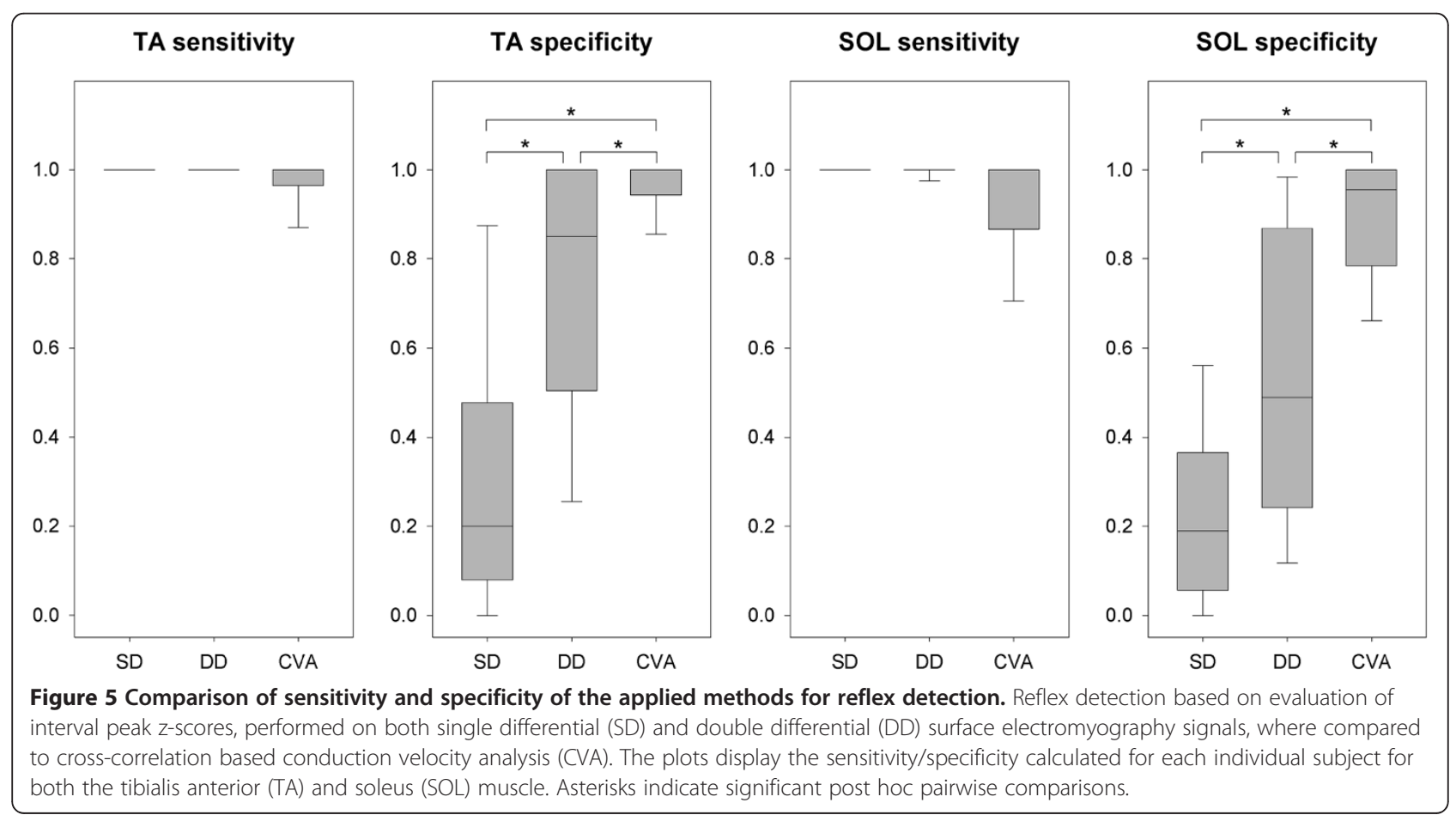


authenticity of any apparent reflex observed as illustrated in Figure 2.

The present study utilized iEMG to obtain an improved gold standard that allows for a distinction between crosstalk and genuine reflexes. The results demonstrated that crosstalk may easily fulfill both the investigated scoring criteria (interval peak z-score $>12$ ) and the criteria for subjective assessment presented by Rhudy and France [4] and France et al. [5]. Consequently, the validation of the interval peak z-score seems limited to the ability to detect ongoing electrophysiological activity. The origin of the electrical signal (the effectuating muscles) is not considered, rendering muscle-specific reflex detection problematic.

\section{Crosstalk implications on reflex detection}

The NWR utilizes complex muscle synergies to effectively withdraw a limb from potential noxious stimuli and will often involve activation of more than one muscle [1]. This may cause a sEMG recording of a reflex response to be a mixture of signals originating from various muscles. Consequently, valid muscle specific reflex detection requires a distinction between signal components originating from adjacent muscles (i.e. crosstalk) and genuine muscle activity in the investigated muscle. This is of particular importance whenever the muscles executing the reflex response are of interest e.g. during mapping of RRF or assessment of RRF modulation for specific muscles in relation to variation in nociceptive sensitivity [22].

This study clearly demonstrated the possible consequences of disregarding crosstalk during reflex detection. The evaluation of interval peak $\mathrm{z}$-scores calculated for SD sEMG signals (the standard recording of reflexes in both experimental and clinical settings) resulted in reflex detection with an extremely poor specificity $(0.20$ and 0.19 for TA and SOL respectively). In this study, nearly half of all detected reflexes were not genuine reflexes but merely reflecting crosstalk. The amount of crosstalk depends on anatomical conditions, such as thickness of the subcutaneous layer $[7,8]$, and clearly on the magnitude of the originating muscle activity.

The amplitude of reflexes in the present study may be modulated in several ways, most effectively by manipulation of stimulation intensities. Consequently, the specificity for reflex detections based on evaluation of interval peak z-scores may be influenced by varying stimulation intensities. However, the stimulation intensities applied in this study (1.2-1.5 times the reflex threshold) were lower than those generally applied in previous studies using similar experimental setups [22,24]. This indicates that the magnitude of crosstalk observed in this study may be representative for NWR assessment using a similar experimental setup and hence that, under certain conditions, crosstalk may pose a serious problem during NWR assessment.
Distinction between crosstalk and genuine muscle activity This study has clearly demonstrated that significantly different muscle fiber CVs may be estimated for genuine reflexes and crosstalk, respectively. The underlying hypothesis has been tested using two standardized methods for CVs estimation based on different types of sEMG. The apparent CVs estimated for crosstalk are unreasonable high from a physiological perspective, reflecting that the main components of the signals are not propagating at all but are observed roughly simultaneously at the two adjacent recording sites. The apparent $\mathrm{CVs}$ estimated due to electrical crosstalk alone were more than one order of magnitude higher than CVs estimated for genuine reflexes, rendering the precision of the simple crosscorrelation technique sufficient to allow distinction between crosstalk and genuine reflexes. However, the limited precision of the cross-correlation technique and the resulting relatively large variability in $\mathrm{CVs}$ estimations did entail detection thresholds for muscle fiber CVs well above the physiological range.

Restrictive inclusion criteria for the individual sweeps recorded has been employed to ensure the existence of two separate datasets consisting exclusively of crosstalk and genuine reflexes, respectively. While appropriate and necessary in order to properly demonstrate the differences in $\mathrm{CV}$ for crosstalk and genuine muscle activity, it leaves room for more complex scenarios, involving both crosstalk and genuine reflex activity within the reflex window for further research. When practical measures are considered, crosstalk may be due to a combination of travelling and non-travelling signal components depending on the distance between the active muscle fibers and the detection point [8]. In the case of a mixture of crosstalk and genuine muscle activity an estimation of $\mathrm{CV}$ will involve a weighted average of the temporal delay of both propagating and non-propagating signal components originating from both crosstalk and genuine muscle activity. Whether estimation of $\mathrm{CV}$ of a recorded signal indeed reflects the amount of crosstalk in a reliable manner needs to be investigated and could potentially allow not only detection but also estimation of crosstalk during reflex detection.

\section{Conduction velocity estimation}

Various $\mathrm{CV}$ estimation techniques differ with respect to specific definitions of the delay between signals which in practice have unequal shapes [25]. Hence, minor discrepancies between CVs estimated using different methods are inevitable. However, the most evident difference between the two sets of muscle fiber $\mathrm{CV}$ estimations is the width of their confidence intervals. $\mathrm{CV}$ estimation using a sophisticated algorithm performed on several sEMG channels recorded using an electrode array had a higher precision than the much simpler cross-correlation technique performed on two SD sEMG channels. This was expected 
and the precise maximum likelihood estimator was intended for validation of estimated CVs and to constitute reference values for future work.

Reliable evaluation of whether a signal is dominated by propagating or non-propagating signals components requires estimation of $\mathrm{CV}$ with a certain degree of accuracy and precision. The simple and convenient method for muscle fiber CV estimation, constituting the core of this new methodology for reflex detection, is best suited for long superficial muscles with parallel fibers like TA. The performance of the novel methodology may therefore vary when applied on different muscles, but even evaluation of a bi-pennate muscle with short, nonparallel fibers like SOL did allow reflex detection with excellent accuracy.

Whereas the CVs estimated for TA were slightly higher than previous findings [16], the CVs estimated for SOL in the present study were definitely higher than physiologically reasonable. However, a considerable overestimation had to be expected considering the rather wide pennation angle for this muscle. SOL has a pennation angle of approximately 25 degrees at rest [26], reducing the effective inter-electrode distance along the orientation of the fibers about $10 \%$, causing an equivalent overestimation of the average $\mathrm{CV}$.

Furthermore, Broman et al. [27] reported CVs up to $8 \mathrm{~m} / \mathrm{s}$ for TA estimated using the applied crosscorrelation technique on SD signals, whereas application of the same technique on DD recordings eliminated these supra-physiological CV estimations. These high CVs may be caused by the existence of both propagating and non-propagating signal components due to inhomogeneity and anisotropic properties of the volume conductor and may possibly be reduced by evaluation of cross-correlations of DD signals instead of SD recording.

Additionally, the high-pass filtering prior to CV estimation using the cross-correlation technique may result in $\mathrm{CV}$ overestimation. The cut-off frequencies constitute a compromise between maintenance of a sufficient high SNR and rejection of distorted waves, and will accordingly differ between the two muscles due to marked difference in muscle fiber length. As such, an optimal cut-off frequency cannot be selected, especially for SOL. The applied cut-off frequency at $100 \mathrm{~Hz}$ attenuates signal components with wavelengths exceeding $40 \mathrm{~mm}$ considering an average $\mathrm{CV}$ of $4 \mathrm{~m} / \mathrm{s}$, whereas the length of SOL muscle fibers at rest are approximately 35-38 $\mathrm{mm}$ [26]. Even under the most unlikely assumption (that the motor end plates are located at the end of the fibers), distorted waves remain. However, increasing the cut-off frequency will result in a SNR that would be too low to allow meaningful $\mathrm{CV}$ estimation.

\section{Applicability and necessity of improved reflex detection}

The validation of the interval peak z-score, based on visual examination of SD sEMG recordings, carried out in this study supports previous findings [4,5]; a threshold value around 12 will allow accurate and reliable detection of apparent reflexes. However, in the presence of crosstalk, not all electrophysiological activity observed represents a genuine reflex involving the muscle investigated. This potential issue was elucidated by the application of a refined gold standard (visual examination of iEMG [28]), allowing distinction between crosstalk and genuine reflexes and resulting in improved validation. It was hereby revealed that application of an interval peak z-score threshold of 12 to achieve muscle specific reflex detection may result in an extremely poor specificity, especially when performed on SD sEMG signals. As shown in the plots of sensitivity and specificity (Figure 4), a strikingly improved specificity combined with a reasonable sensitivity could be achieved by setting a much higher threshold for the interval peak z-score. Evaluation of interval peak z-scores calculated for DD sEMG signals may allow a joint value of sensitivity and specificity of 0.95 if the threshold was set at 48 instead of 12 .

It is nevertheless stressed that one optimal, fixed threshold for the interval peak z-score cannot be established, and that custom thresholds should be chosen with great care. The application of a very high threshold in order to distinguish genuine reflexes from crosstalk based on the magnitude of the electrophysiological measurements would work well on this specific dataset. However, this will probably not be the general case. Both the optimal interval peak $\mathrm{z}$-score threshold and the resulting sensitivity and specificity may vary strongly depending on the data in question. In any case, sufficiently small reflexes will be mistaken for crosstalk and erroneously undetected. Since experimental and clinical protocols often emphasize evaluation of reflex thresholds, this poses a serious problem. This problem does not arise when applying CVA for reflex detection, which constitutes a major advantage of this novel methodology.

The optimal method for reflex detection depends on specific challenges and requirements, including the presence of crosstalk and also weighting of sensitivity and specificity. Reflex detection based on evaluation of interval peak z-scores performed on both SD and DD sEMG entailed perfect sensitivity, indicating great performance in the absence of crosstalk. However, in the presence of crosstalk, the evaluation of DD sEMG instead of SD signals may entail a significant improvement in detection accuracy. This is clear from the plots of sensitivity and specificity (Figure 4) where both sensitivity and specificity, for all interval peak z-score thresholds, are superior for evaluation of DD sEMG compared to SD sEMG. The statistical analysis and the box-plots in Figure 5 suggest 
that CVA seems to entail slightly lowered sensitivity, especially for SOL. Thus, the relative value of sensitivity and specificity respectively ought to be weighted prior to deciding whether to apply the novel methodology or to purely evaluate interval peak z-scores calculated for DD sEMG. Also the risk and magnitude of crosstalk should be considered. In cases with seldom and weak crosstalk, the specificity achieved by reflex detection based on evaluation of interval peak z-scores calculated for DD sEMG may be sufficient, rendering superior sensitivity. However, whenever muscle specific reflex detection with a reliable high specificity is required, CVA should be seriously considered.

\section{Beyond reflex detection}

CVA may be viewed as an additional binary evaluation following another reflex detection methodology, in order to assess whether a detected reflex indeed is a genuine reflex or merely the result of crosstalk. There seems to be no reason why this approach should be less efficient detecting crosstalk during static or voluntary contractions. Hence, this paper presents a convenient generic method for qualitative assessment of crosstalk, applicable on signals recorded using standard sEMG equipment and procedures which may possibly be utilized to ensure a more specific and reliable detection of genuine muscle activation e.g. during gait analysis, biofeedback therapy, prosthetic control, or other applications.

\section{Conclusion}

This study demonstrated possible consequences of disregarding electrical crosstalk during reflex detection from sEMG signals. Evaluation using a previously validated scoring criterion (interval peak z-score) calculated for standard SD sEMG signals resulted in reflex detection with poor specificity due to crosstalk. In the presence of crosstalk, the evaluation of DD sEMG instead of SD signals may entail a significant improvement in detection accuracy. Furthermore, this study demonstrated that significantly different muscle fiber CVs may be estimated for genuine reflexes and crosstalk, respectively. A novel methodology, CVA, was developed to allow reliable detection of EMG crosstalk during reflex detection, which resulted in reflex detection with excellent sensitivity and specificity.

\footnotetext{
Abbreviations

CV: Conduction velocity; CVA: Conduction velocity analysis; DD: Double differential; iEMG: Intramuscular electromyography; NWR: Nociceptive withdrawal reflex; RRF: Reflex receptive field; SD: Single differential; sEMG: Surface electromyography; SNR: Signal-to-noise ratio; SOL: Soleus; TA: Tibialis anterior.
}

\section{Competing interests}

The authors declare that they have no competing interests.

\section{Authors' contributions}

MBJ, JBM and OKA defined the research topic. MBJ and KSF designed the experiment and carried out the data acquisition. MBJ analyzed the data, developed the presented methodology, interpreted the results and wrote the paper. JBM and OKA have contributed to the analysis, interpretation, and presentation of the paper. All authors have read and approved the final manuscript.

Received: 30 October 2012 Accepted: 21 March 2013

Published: 26 March 2013

\section{References}

1. Sandrini G, Serrao M, Rossi P, Romaniello A, Cruccu G, Willer JC: The lower limb flexion reflex in humans. Prog Neurobiol 2005, 77(6):353-395.

2. Skljarevski $V$, Ramadan NM: The nociceptive flexion reflex in humans review article. Pain 2002, 96(1-2):3-8.

3. Lim EC, Sterling M, Stone A, Vicenzino B: Central hyperexcitability as measured with nociceptive flexor reflex threshold in chronic musculoskeletal pain: a systematic review. Pain 2011, 152(8):1811-1820.

4. Rhudy $\lrcorner$, France $C R$ : Defining the nociceptive flexion reflex (NFR) threshold in human participants: a comparison of different scoring criteria. Pain 2007, 128(3):244-253.

5. France $C R$, Rhudy JL, McGlone S: Using normalized EMG to define the nociceptive flexion reflex (NFR) threshold: further evaluation of standardized NFR scoring criteria. Pain 2009, 145(1-2):211-218.

6. De Luca CJ, Merletti R: Surface myoelectric signal cross-talk among muscles of the leg. Electroencephalogr Clin Neurophysiol 1988, 69(6):568-575.

7. Farina D, Merletti R, Indino B, Graven-Nielsen T: Surface EMG crosstalk evaluated from experimental recordings and simulated signals. Reflections on crosstalk interpretation, quantification and reduction. Methods Inf Med 2004, 43(1):30-35.

8. Mesin L, Smith S, Hugo S, Viljoen S, Hanekom T: Effect of spatial filtering on crosstalk reduction in surface EMG recordings. Med Eng Phys 2009, 31(3):374-383.

9. Dimitrova NA, Dimitrov GV, Nikitin OA: Neither high-pass filtering nor mathematical differentiation of the EMG signals can considerably reduce cross-talk. J Electromyogr Kinesiol 2002, 12(4):235-246.

10. Farina D, Merletti R, Indino B, Nazzaro M, Pozzo M: Surface EMG crosstalk between knee extensor muscles: experimental and model results. Muscle Nerve 2002, 26(5):681-695.

11. Farina D, Arendt-Nielsen L, Merletti R, Indino B, Graven-Nielsen T: Selectivity of spatial filters for surface EMG detection from the tibialis anterior muscle. IEEE Trans Biomed Eng 2003, 50(3):354-364.

12. Frahm KS, Jensen MB, Farina D, Andersen OK: Surface EMG crosstalk during phasic involuntary muscle activation in the nociceptive withdrawal reflex. Muscle Nerve 2012, 46(2):228-236.

13. Farina D, Muhammad W, Fortunato E, Meste O, Merletti R, Rix H: Estimation of single motor unit conduction velocity from surface electromyogram signals detected with linear electrode arrays. Med Biol Eng Comput 2001, 39(2):225-236.

14. Arendt-Nielsen L, Mills KR: Muscle fibre conduction velocity, mean power frequency, mean EMG voltage and force during submaximal fatiguing contractions of human quadriceps. Eur J Appl Physiol Occup Physiol 1988, 58(1-2):20-25.

15. Arendt-Nielsen L, Mills KR: The relationship between mean power frequency of the EMG spectrum and muscle fibre conduction velocity. Electroencephalogr Clin Neurophysiol 1985, 60(2):130-134.

16. Broman H, Bilotto G, De Luca CJ: Myoelectric signal conduction velocity and spectral parameters: influence of force and time. J Appl Physiol 1985, 58(5):1428-1437.

17. Schouenborg J, Kalliomaki J: Functional organization of the nociceptive withdrawal reflexes. I. Activation of hindlimb muscles in the rat. Exp Brain Res 1990, 83(1):67-78.

18. Weng HR, Schouenborg J: Cutaneous inhibitory receptive fields of withdrawal reflexes in the decerebrate spinal rat. J Physiol 1996, 493(Pt 1):253-265.

19. Schouenborg J: Modular organisation and spinal somatosensory imprinting. Brain Res Brain Res Rev 2002, 40(1-3):80-91.

20. Clarke RW, Harris J: The organization of motor responses to noxious stimuli. Brain Res Brain Res Rev 2004, 46(2):163-172.

21. Sonnenborg FA, Andersen OK, Arendt-Nielsen L, Treede RD: Withdrawal reflex organisation to electrical stimulation of the dorsal foot in humans. Exp Brain Res 2001, 136(3):303-312.

22. Andersen OK, Sonnenborg FA, Arendt-Nielsen L: Modular organization of human leg withdrawal reflexes elicited by electrical stimulation of the foot sole. Muscle Nerve 1999, 22(11):1520-1530. 
23. Sonnenborg FA, Andersen OK, Arendt-Nielsen L: Modular organization of excitatory and inhibitory reflex receptive fields elicited by electrical stimulation of the foot sole in man. Clin Neurophysiol 2000, 111(12):2160-2169.

24. Neziri AY, Curatolo M, Bergadano A, Petersen-Felix S, Dickenson A, ArendtNielsen L, Andersen OK: New method for quantification and statistical analysis of nociceptive reflex receptive fields in humans. $J$ Neurosci Methods 2009, 178(1):24-30.

25. Farina D, Merletti R: Methods for estimating muscle fibre conduction velocity from surface electromyographic signals. Med Biol Eng Comput 2004, 42(4):432-445

26. Maganaris CN, Baltzopoulos V, Sargeant AJ: In vivo measurements of the triceps surae complex architecture in man: implications for muscle function. J Physiol 1998, 512(Pt 2):603-614.

27. Broman H, Bilotto G, De Luca CJ: A note on the noninvasive estimation of muscle fiber conduction velocity. IEEE Trans Biomed Eng 1985, 32(5):341-344

28. van Vugt JP, van Dijk JG: A convenient method to reduce crosstalk in surface EMG. Cobb Award-winning article 2001. Clin Neurophysiol 2001, 112(4):583-592.

doi:10.1186/1471-2202-14-39

Cite this article as: Jensen et al:: Analysis of muscle fiber conduction velocity enables reliable detection of surface EMG crosstalk during detection of nociceptive withdrawal reflexes. BMC Neuroscience 2013 14:39.

\section{Submit your next manuscript to BioMed Central and take full advantage of:}

- Convenient online submission

- Thorough peer review

- No space constraints or color figure charges

- Immediate publication on acceptance

- Inclusion in PubMed, CAS, Scopus and Google Scholar

- Research which is freely available for redistribution 